\title{
The Angelman Syndrome Protein Ube3a Is Required for Polarized Dendrite Morphogenesis in Pyramidal Neurons
}

\author{
Sheng Miao, ${ }^{1}$ Renchao Chen, ${ }^{1}$ Jiahao Ye, ${ }^{1}$ Guo-He Tan, ${ }^{1}$ Shuai Li, ${ }^{1}$ Jing Zhang, ${ }^{1}$ Yong-hui Jiang, ${ }^{2}$ and Zhi-Qi Xiong ${ }^{1}$ \\ ${ }^{1}$ Institute of Neuroscience and State Key Laboratory of Neuroscience, Shanghai Institutes for Biological Sciences, Chinese Academy of Sciences, Shanghai \\ 200031, China and 2Division of Medical Genetics, Department of Pediatrics, Duke University School of Medicine, Durham, North Carolina 27710
}

Pyramidal neurons have a highly polarized dendritic morphology, characterized by one long apical dendrite and multiple short basal dendrites. They function as the primary excitatory cells of the mammalian prefrontal cortex and the corticospinal tract. However, the molecular mechanisms underlying the development of polarized dendrite morphology in pyramidal neurons remain poorly understood. Here, we report that the Angelman syndrome (AS) protein ubiquitin-protein ligase E3A (Ube3a) plays an important role in specifying the polarization of pyramidal neuron dendritic arbors in mice. shRNA-mediated downregulation of Ube3a selectively inhibited apical dendrite outgrowth and resulted in impaired dendrite polarity, which could be rescued by coexpressing mouse Ube3a isoform 2, but not isoform 1 or 3. Ube3a knockdown also disrupted the polarized distribution of the Golgi apparatus, a well established cellular mechanism for asymmetric dendritic growth in pyramidal neurons. Furthermore, downregulation of Ube3a completely blocked Reelin-induced rapid deployment of Golgi into dendrite. Consistently, we also observed selective inhibition of apical dendrite outgrowth in pyramidal neurons in a mouse model of AS. Overall, these results show that Ube3a is required for the specification of the apical dendrites and dendrite polarization in pyramidal neurons, and suggest a novel pathological mechanism for AS.

\section{Introduction}

Dendritic morphology varies greatly according to neuronal cell type, and specific morphological characteristics are linked with various functional implications (Jan and Jan, 2010). Pyramidal neurons, for example, are the primary excitatory neuronal cell type in the cerebral cortex, the hippocampus and the amygdala, and are characterized by their polarized dendritic shape. They have a long apical dendrite that extends hundreds of micrometers toward the pia as well as several smaller and shorter basolateral dendrites (Whitford et al., 2002). The distinct structures of these two types of dendrites determine their specific functions in receiving and integrating spatial information. However, the exact molecular mechanisms underlying the development of highly polarized dendrite morphology in pyramidal neurons remain unclear.

The gene UBE3A encodes a HECT domain ubiquitin E3 ligase and its deficiency in humans is primarily responsible for Angelman syndrome (AS), a neurodevelopmental disorder characterized by developmental delay, intellectual disability,

\footnotetext{
Received May 24, 2012; revised Sept. 28, 2012; accepted 0ct. 30, 2012.

Author contributions: S.M. and Z.-Q.X. designed research; S.M., R.C., J.Y., G.-H.T., S.L., and J.Z. performed research; S.M., R.C., and Y.-h.J. contributed unpublished reagents/analytic tools; S.M., R.C., and S.L. analyzed data; S.M. wrote the paper.

This work was supported by grants from the 973 Program (2011CBA00400), the National Natural Science Foundation of China (30925016, 31123002, 31021063), and the Chinese Academy of Sciences (XDA01020305). We thank Dr. Bin Lu for his technical help and Dr. Qian Hu for his help in imaging.

Correspondence should be addressed to Dr. Zhi-Qi Xiong, Institute of Neuroscience and State Key Laboratory of Neuroscience, Shanghai Institutes for Biological Sciences, Chinese Academy of Sciences, 320 Yueyang Road, Shanghai 200031, China. E-mail: xiongzhiqi@ion.ac.cn.

DOI:10.1523/JNEUROSCI.2509-12.2013

Copyright $\odot 2013$ the authors $\quad 0270-6474 / 13 / 330327-07 \$ 15.00 / 0$
}

movement disorder, and epilepsy (Mabb et al., 2011). Although defects in synaptic development and plasticity have been observed in neurons lacking Ube3a protein (Jiang et al., 1998; Dindot et al., 2008; Yashiro et al., 2009; Greer et al., 2010; Margolis et al., 2010), it remains unclear whether earlier developmental events are also regulated by Ube3a. Several lines of evidence support an in-depth study of the role of Ube3a in early stages of brain development, particularly in dendrite morphogenesis. First, observations of microcephaly in an AS patient (Jay et al., 1991) and in an AS mouse model (Jiang et al., 1998) strongly suggest the presence of other developmental abnormalities in addition to synaptic defects. Second, the inhibition of dendritic arborization in the sensory neurons of Drosophila Ube3a-null mutants (Lu et al., 2009) suggests a conserved function of Ube3a in dendrite morphogenesis in mammalian neurons.

In this study, we used RNAi technique to knock down Ube3a expression in developing pyramidal neurons in mouse brain, to determine its function in early neural development events. Our results indicate that Ube $3 \mathrm{a}$ is critical for polarized dendrite morphogenesis in pyramidal neurons and that this role is primarily mediated via mouse Ube3a isoform 2.

\section{Materials and Methods}

Mice and in utero electroporation. The use of mice was in accord with the Society for Neuroscience guidelines and Institutional Animal Care and Use Committee-approved protocols. Ube3a-shRNA or scrambled control shRNA (Sh-scr) was cotransfected with EGFP plasmids into the neural epithelium of the lateral ventricle of timed-pregnant ICR mice using an in utero electroporation method described previously (Saito and $\mathrm{Na}-$ 
katsuji, 2001). For morphological analysis of pyramidal neurons in AS mice, P8 mice of either sex and adult male mice were used.

Mouse Ube3a cloning and construct preparation. Mouse Ube3a cDNAs (GenBank accession numbers NM_001033962, NM_011668 and NM_173010) were cloned from a mouse cerebral cortex cDNA library. PCR products were cloned into the pCAG-IRES-EGFP vector provided by Dr. Xiao-bing Yuan (Institute of Neuroscience and State Key Laboratory of Neuroscience, Shanghai, China) (Zheng et al., 2012). Oligonucleotides targeting different sequences of mouse Ube3a cDNA and a scrambled control oligonucleotide were designed, synthesized, and cloned into pSuper-basic (Oligoengine). The $19 \mathrm{bp}$ target sequences were as follows: Sh-1, 5' -GCC GAA ATC TAG TGA ATG A-3'; Sh-2, 5' ${ }^{\prime}$-CCT ACA AAG TCA TAA GCA A-3'; Sh-scr, 5' -TTC TCC GAA CGT GTC ACG T-3'. The Sh-1-resistant forms of Ube3a were generated by mutating five nucleotides within the Sh-1 targeting sequence in each of the three Ube3a isoforms (from GC CGA AAT CTA GTG AAT GA to GC CG $\underline{G}$ AA $\underline{C}$ CT $\underline{G}$ GT $\underline{A}$ AA $\underline{C}$ GA) without altering the corresponding amino acids. All constructs were verified by sequencing.

Immunohistochemistry. Mice were killed at postnatal day 3 (P3) or P7. Their brains were removed and fixed overnight. After sequential dehydration in $15 \%$ and $30 \%$ sucrose at $4^{\circ} \mathrm{C}, 50 \mu \mathrm{m}$ coronal brain sections were cut with a cryostat, fixed in $4 \%$ PFA for $20 \mathrm{~min}$ at $4^{\circ} \mathrm{C}$, washed three times with $1 \times$ PBS, blocked with $5 \%$ BSA and $0.3 \%$ Triton X-100 in $1 \times$ $\mathrm{PBS}$ for $1 \mathrm{~h}$ at room temperature, and then incubated with rabbit antiGFP $\left(1: 1000\right.$, Invitrogen) primary antibody overnight at $4^{\circ} \mathrm{C}$. The rinsed sections were then incubated with Alexa 488-conjugated goat anti-rabbit secondary antibody (1:3000; catalog \#A-11122; Invitrogen) for $2 \mathrm{~h}$ at $37^{\circ} \mathrm{C}$. For endogenous Ube3a and GM130 immunostaining, sections were heated to $92^{\circ} \mathrm{C}$ for $20 \mathrm{~min}$ in $1 \times$ SSC buffer, pH 6.0, for antigen retrieval before the blocking step. The sections were then incubated with anti-Ube3a (1:3000; catalog \#E8655; Sigma) or anti-GM130 antibody (1:1000; catalog \#610822; BD Biosciences).

Analysis of dendrite orientation. The orientation of each dendrite was measured as previously described (Demyanenko et al., 2004) using Image-Pro Plus 6.0 software. Briefly, a $10-\mu \mathrm{m}$-radius circle was drawn around the soma center. The circle crossed each dendrite at individual point. A line was then drawn from the center of the soma through the crossing point of the dendrite on the circle. The angle made by this line relative to a line drawn from the soma perpendicular to the pial surface was expressed in degrees.

Image acquisition and quantification. Images were obtained with a Nikon A1R inverted confocal microscope with $20 \times$ (numerical aperture, 0.7 ) or $63 \times$ (numerical aperture, 1.4) objectives. Each image was a composite constructed from a series of images taken throughout the $z$ aspect of the neuron. Neuronal morphology was traced and analyzed with Neurolucida software (MBF Bioscience).

Statistical analysis. Values are expressed as mean \pm SEM. Student's $t$ test was used to measure significance of differences between two groups. Statistical significance was defined as $p<0.05$.

\section{Results \\ Downregulation of Ube3a disrupts dendrite polarity in pyramidal neurons}

To investigate the role of Ube3a in neuronal development, we downregulated its expression in cortical pyramidal neurons using shRNA constructs delivered via in utero electroporation at embryonic day 13.5 (E13.5). Expression of two shRNA vectors, shRNA-1 and 2 (Sh-1 and -2), markedly reduced the expression of native Ube 3 a in cortical neurons (Fig. $1 A, B$ ). To assess the impact of Ube3a knockdown on pyramidal neurons, a morphological analysis was performed on layer II/III cortical neurons of the somatosensory cortex 1 (S1) at P7, which corresponds to a period of robust dendrite morphogenesis. Most Sh-scr-transfected neurons displayed a typical polarized dendritic arbor (Fig. 1C). In contrast, many neurons transfected with either Sh-1 or Sh-2 exhibited no obvious apical dendrite, although basal dendrite-like processes in these neurons appeared normal.

We next defined and quantified dendrite polarity based on two morphological features specific to the apical dendrite: its vertical orientation toward the pial surface and its length, as it is the longest among all primary dendrites. We started by quantifying dendrite polarity according to the orientation property of the apical dendrite. First, the angle of each dendrite relative to the line perpendicular to the pial surface was measured in Sh-scrtransfected neurons. Consistent with previous studies (Sasaki et al., 2002; Demyanenko et al., 2004), apical dendrites in these neurons were mostly distributed within the range of $\pm 15^{\circ}$, whereas most basal dendrites were outside the range of $\pm 40^{\circ}$. Therefore, we defined normal dendrite polarity as a single dendrite within the $\pm 15^{\circ}$ range and the rest outside the $\pm 40^{\circ}$ range (Fig. 1D). The vast majority of neurons transfected with Sh-scr exhibited normal polarized dendrite morphology, whereas a significant portion of neurons expressing either Sh-1 or Sh-2 showed defects in dendrite polarity at P7 (Fig. 1E).

Next, we quantified dendrite polarity in transfected neurons based on the length property of the apical dendrite. We designated the longest dendrite as the apical dendrite and the rest as basal dendrites, and used two polarity indexes to compare them: apical/mean basal, defined as the ratio of apical dendrite length to basal dendrite mean length, and apical/total dendrite branch length (TDBL), defined as the ratio of apical dendrite length to total dendrite branch length. We found that apical, but not basal, dendrite length was markedly reduced in shRNA-treated neurons relative to control neurons, as were the values of both polarity indexes (Fig. $1 F-J$ ).

To explore the timing and mechanism of dendritic polarity impairment in Ube3a-shRNA-transfected neurons, we analyzed the morphology of layer II/III cortical neuron dendrites earlier in development at $\mathrm{P} 3$, when radial migration has completed and dendrites begin to elaborate into an arborized tuft (Tabata and Nakajima, 2002). We found that dendrite length and polarity indexes were comparable between both shRNAs and control groups at P3 (Fig. $1 E-J$ ). These findings indicate that the dendrite polarization defects observed in P7 neurons treated with Ube3a-shRNA results from selective inhibition of apical dendrite outgrowth after P3, rather than a general stalling of dendrite growth.

\section{The role of Ube3a in polarized dendrite morphogenesis is primarily mediated via mouse Ube3a isoform 2}

To date, three Ube3a isoforms have been identified in mouse (Greer et al., 2010). Isoform 2 (Iso2) corresponds to the open reading frame of Ube3a, while Iso 1 and Iso3 both lack 21 aa from their $\mathrm{N}$ termini. Iso 1 also lacks 87 aa from its $\mathrm{C}$ terminus, a region that is critical for hect domain function; Isol has therefore been proposed to be an E3 ligase deficient form. However, the expression profile of each Ube3a isoform in developing cortex has not yet been well characterized. We examined the protein levels of these three isoforms from total lysate of developing cortex using antibody which could recognize all three isoforms. Interestingly, only one band, which might corresponded to Iso2 $(\sim 95 \mathrm{kDa})$ or Iso3 ( $\sim 94 \mathrm{kDa})$ or both, could be detected from E15 to adult (Fig. $2 A)$. The band was maintained through embryogenesis and early postnatal life with a slight decline after P7. The Isol $(\sim 84 \mathrm{kDa})$ corresponding band could not be detected probably due to low level of expression. Further, we quantified the mRNA level of each isoform with Real-time PCR and confirmed that Isol is expressed at low level in the developing cortex (Fig. 2B). To de- 
A

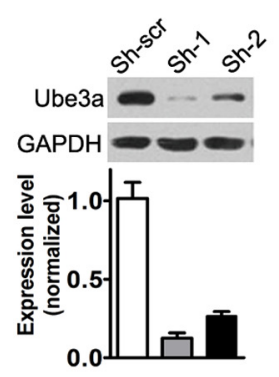

B

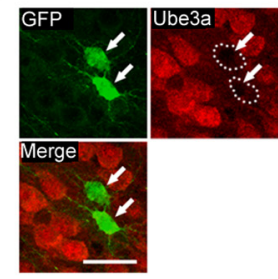

G

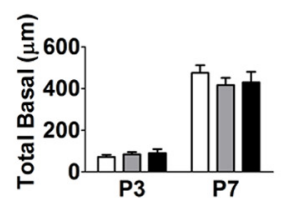

C
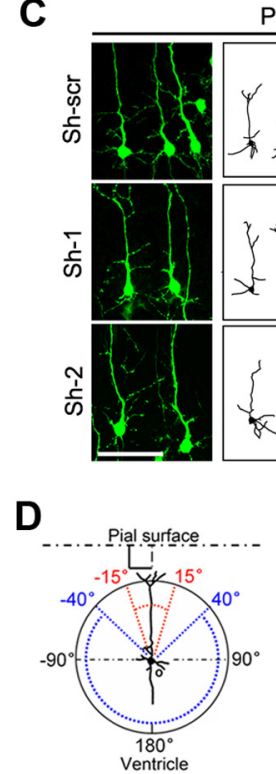

H

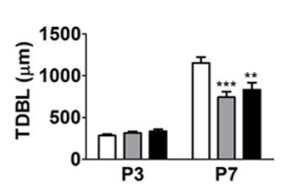

P3

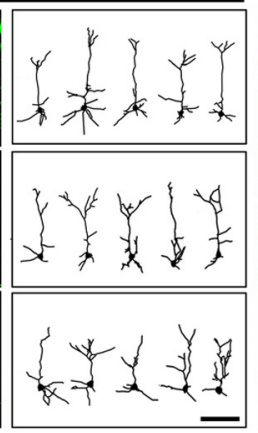

E

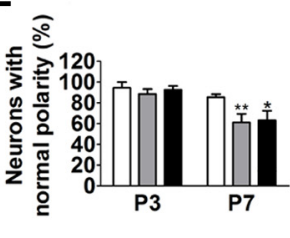

I

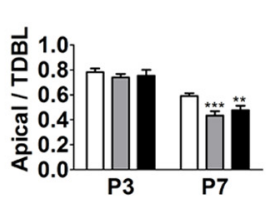

P7

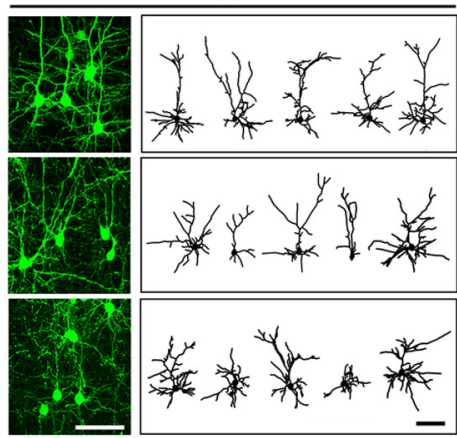

$\mathbf{F}$

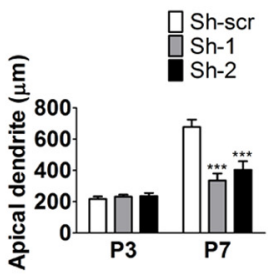

J

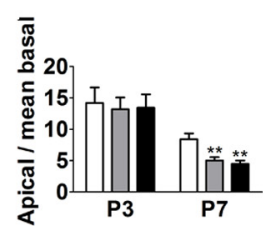

Figure 1. Ube3a knockdown selectively inhibits apical dendrite outgrowth, thereby decreasing dendrite polarity. $\boldsymbol{A}$, The top shows a Ube3a Western blot of lysates from DIV 5 cultured cortical neurons transfected at dissociation with the indicated shRNA constructs. GAPDH was used as a loading control. Quantification of these results is shown in the bottom. $\boldsymbol{B}$, Immunohistochemical staining of native Ube3a in coronal sections from a P7 mouse brain electroporated with Sh-1 and GFP at E13.5. The white arrows indicate successfully transfected cells. Scale bar, $25 \mu \mathrm{m}$. $C$, Representative images and tracings of layer II/III pyramidal neurons from P3 and P7 mouse brains treated with the indicated constructs. Scale bar, $50 \mu \mathrm{m}$. D, Schematic representation of the methodology used to determine dendritic polarity, based on the orientation properties of the two types of dendrite relative to the pial surface. $E$, Graphical representation of the percentage of labeled neurons with normal dendritic polarity. $n>4$ brains in each group. $\boldsymbol{F}-J$, Quantitative analyses of apical dendrite length $(\boldsymbol{F})$, total length of basal dendrites $(\boldsymbol{G}), \operatorname{TDBL}(\boldsymbol{H})$, the ratio of apical dendrite length to TDBL $(I)$, and the ratio of apical dendrite length to basal dendrite mean length $(\boldsymbol{J})$. Data represent mean \pm SEM. $n=20-50$ neurons in each group. ${ }^{*} p<0.05 ;{ }^{* *} p<0.01 ;{ }^{* * *} p<0.001$; statistically significant differences were determined using $t$ test.

termine the particular role of each isoform in dendrite polarization in cortical pyramidal neurons, we conducted a series of rescue experiments. Plasmids encoding the shRNA-resistant form of each isoform (Fig. 2E) were cotransfected together with Sh-1-expressing plasmids using in utero electroporation at E13.5. Immunostaining for Ube3a revealed distinct patterns of subcellular localization of the overexpressed isoforms in P7 cortical pyramidal neurons (Fig. 2D). Isol and 2 were primarily distributed in the cytosol and dendrites, whereas Iso3 appeared to be confined largely to the nucleus. Interestingly, the impaired dendrite polarity caused by Ube3a knockdown was specifically rescued by coexpression of the shRNA-resistant Iso2 (Iso2R) (Fig. $2 F-H)$. By comparison, both the ligase-dead cytoplasmic Iso1R and the nuclear-located Iso3R were ineffective in restoring dendrite polarity. Consistent with these findings, in immature DIV4 or P7 neurons, when neuronal morphogenesis is just in progress, we found that Ube3a was localized primarily in the cytosol of cell bodies and processes, whereas in more mature DIV12 or P30 neurons, Ube3a was highly enriched in the nucleus (Fig. 2C). Notably, high levels of Ube3a were detected in apical dendrites close to the soma (white arrow in Fig. 2C), but not in the basal dendrites of P7 neurons. These results indicate that E3 ligase activity and cytoplasmic localization of Ube3a are both required for its role in dendrite polarization, and suggest that Ube3a regulates this process by modulating its cytoplasmic substrates. Notably, overexpression of Iso2 had no significant effect on dendritic morphogenesis (Fig. $2 \mathrm{~F}-H$ ).
In addition to the cerebral cortex, pyramidal neurons are also found in the CA1 region of the hippocampus. These CA1 neurons undergo similar developmental events to cortical neurons before reaching maturation (Nakahira and Yuasa, 2005). Here, we found that CA1 neurons transfected with Ube3a-shRNA exhibited defects in dendrite morphology and polarity comparable to those observed in cortical neurons (Fig. 3C). These findings indicate that Ube3a plays an important role in the development of dendrite polarity, in both cortical and hippocampal pyramidal neurons.

\section{Ube3a knockdown disrupts the polarized distribution of the} Golgi apparatus in pyramidal neurons

As a crucial component of the secretory pathway, the Golgi apparatus is distributed in a polarized fashion in pyramidal neurons, being preferentially located on the apical side of the soma and extending into the apical dendrite (Horton et al., 2005). This asymmetric distribution appears to be responsible for the preferential trafficking of membranous cargos into the apical dendrite to support polarized dendrite morphogenesis (Horton et al., 2005). To determine the effect of Ube3a knockdown on the localization of the Golgi apparatus, we examined Golgi morphology in neocortical and hippocampal pyramidal neurons by immunostaining for GM130, a cis-Golgi protein marker. In P3 and $\mathrm{P} 7$ cortical neurons and $\mathrm{P} 7$ hippocampal neurons transfected with Sh-scr, the Golgi apparatus was enriched on the apical side 
and in the proximal region of the apical dendrite (Fig. $3 A, D$ ). By comparison, in the Ube3a-shRNA-transfected neurons at P3 and P7, the Golgi apparatus often appeared clustered near the nucleus rather than extending into a dendrite (Fig. $3 A, D)$. Furthermore, the distance from the center of the nucleus to the tip of the Golgi ribbon was significantly reduced in Ube3a-shRNA-transfected neurons compared with control neurons (Fig. $3 B, E$ ). These results provide in vivo evidence supporting a functional association between the asymmetrical localization of the Golgi apparatus and polarized dendrite morphology. They also suggest that Ube3a may regulate dendrite polarity by modulating the distribution of the Golgi apparatus.

Reelin-Dab1 signaling has been identified as a critical mechanism in regulating Golgi deployment into dendrite (Matsuki et al., 2010). Reelin treatment for $30 \mathrm{~min}$ was sufficient to drive Golgi into dendrite. To examine whether downregulation of Ube3a could block Reelin-induced rapid dendritic Golgi deployment, we treated Ube3a-shRNA neurons with recombinant Reelin and found that Reelin treatment could no longer drive the Golgi into dendrite (Fig. $3 F-H$ ). Additionally, downregulation of Ube3a markedly inhibited Golgi deployment into dendrite (Fig. $3 F-H$ ). These results suggest that Ube3a is required for Reelin's function in dendritic Golgi deployment.

\section{Reduced pyramidal neuron dendrite polarity in a mouse model of AS}

A previously developed mouse model of AS with maternal Ube3a gene deficiency $(\mathrm{m}-/ \mathrm{p}+)$ recapitulates the major features of human AS with symptoms of motor dysfunction, inducible seizures and context-dependent learning deficits (Jiang et al., 1998). To determine whether dendrite polarity was also impaired in these mice, acute brain slices from P8 and 3.5-month-old AS mice were prepared, and individual neurons were patched and filled with the smallmolecular-weight dye biocytin and visualized with an avidin-conjugated fluorophore. The morphological characteristics of these neurons were subsequently imaged and analyzed. Due to their relatively homogeneous nature and ease of identification compared with cortical neurons, we chose to assess CA1 pyramidal neurons. Similar to Ube3a-shRNA neurons in cortical layer II/III, CA1 neurons in P8 and adult AS mice both displayed stunted apical dendrites compared with their wild-type littermates (Fig. $4 A, C$ ). The values of polarity indexes were also significantly reduced in AS mice relative to WT controls (Fig. 4E-G).
A

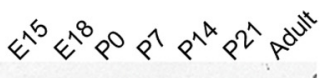
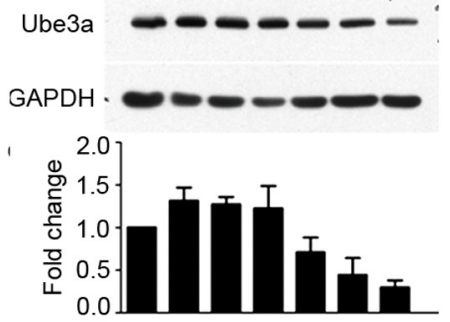

C
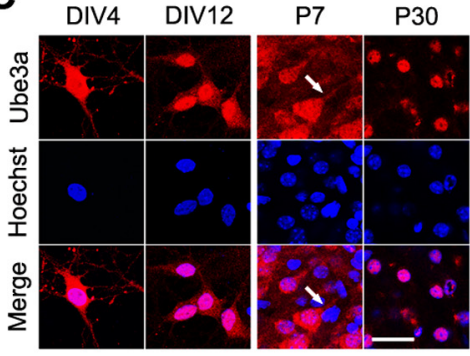

D
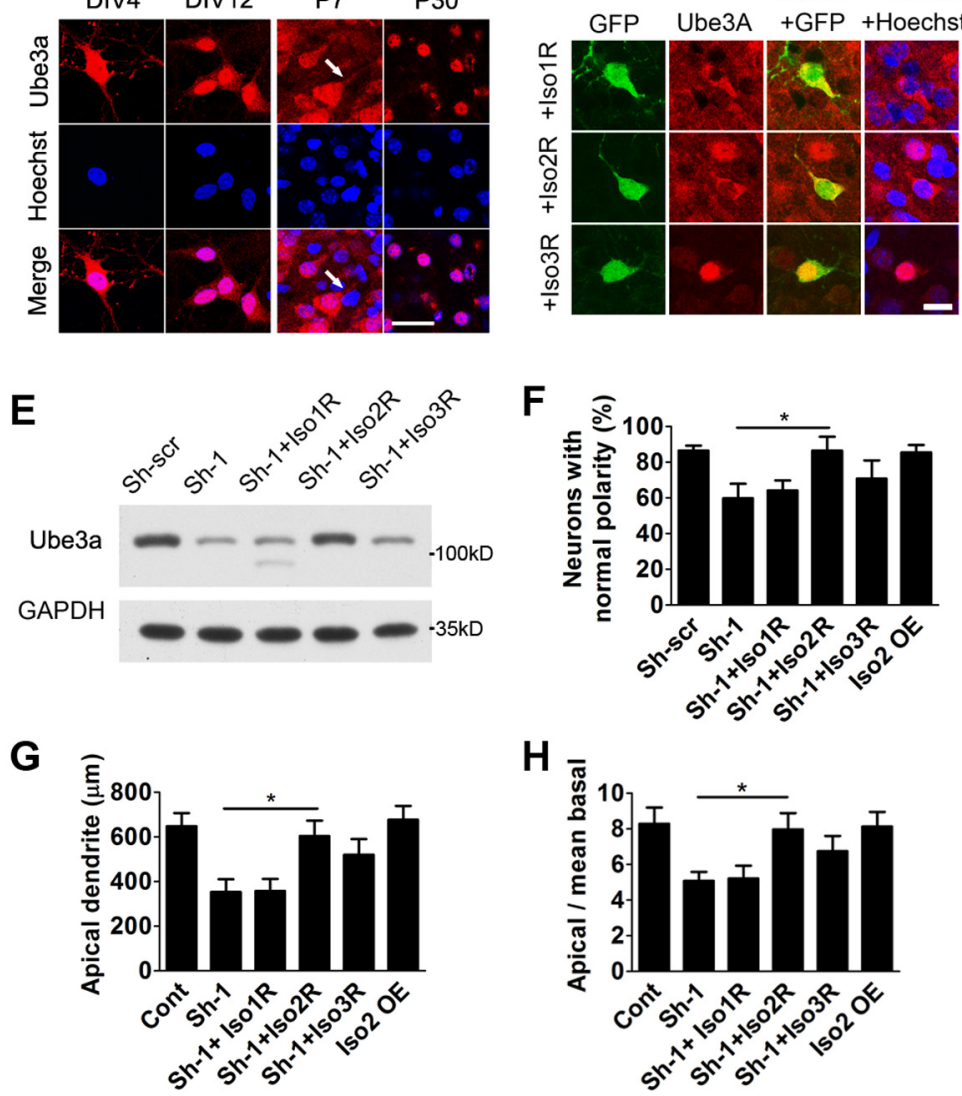

$\mathrm{H}$

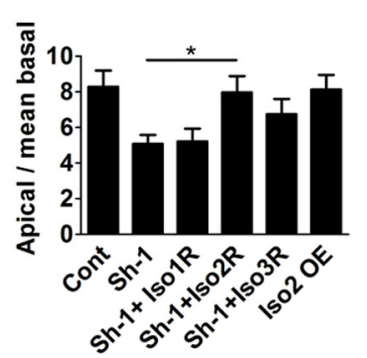

Figure 2. Coexpression of shRNA-resistant mouse Ube3a isoform 2 restores dendrite polarity. $A$, Immunoblot analysis of Ube 3 a expression in the developing cortex. Quantitative analysis is shown in the bottom. $\boldsymbol{B}, \mathrm{qPCR}$ analysis of three isoforms of mouse Ube3a in developing cortex. Their transcript level is normalized to GAPDH. C, Native Ube3a immunostaining (red) and nuclear Hoechst 33342 staining (blue) illustrate the dramatic change in Ube3a subcellular localization, from mostly cytoplasmic in DIV 4 and P7 neurons to nuclear enrichment in DIV12 and P30 neurons. The white arrows point to the proximal region of the apical dendrite. Scale bar, $20 \mu \mathrm{m}$. D, Immunohistochemical Ube3a staining of coronal sections from P7 mouse brains transfected with the indicated constructs. Scale bar, $10 \mu \mathrm{m}$. $\boldsymbol{E}$, Immunoblot of lysates from DIV 5 cultured cortical neurons transfected at dissociation with the indicated constructs. $\boldsymbol{F}$, Quantitative analysis of the percentage of neurons with normal polarized dendrite morphology in layer II/III. $n=5$ brains in each group, and $>100$ neurons for each groups were quantified. $\mathbf{G}, \boldsymbol{H}$, Quantitative analyses of apical dendrite length $(\boldsymbol{G})$ and apical/mean basal ratio $(\boldsymbol{H})$. Data represent mean \pm SEM. $n>20$ neurons in each group. ${ }^{*} p<0.05$; statistically significant differences were determined using $t$ test.

\section{Discussion}

In the present study, we identify a novel role for Ube3a in polarized dendrite morphogenesis in pyramidal neurons. One important finding is that the loss of Ube3a had differential effects on the two types of pyramidal neuron dendrites, inhibiting the outgrowth of the apical dendrite, but not of basal dendrites. Pyramidal neurons transfected with Ube3a-shRNA did not develop these symptoms right from the onset, since dendritic arbors at P3 were comparable between control and shRNA-treated groups. It 
A

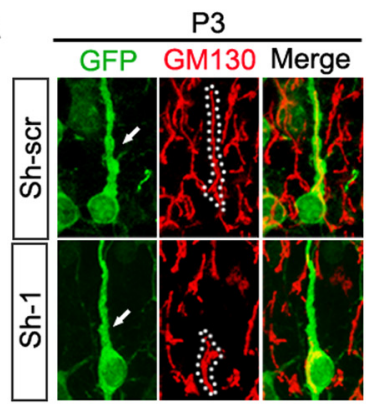

C

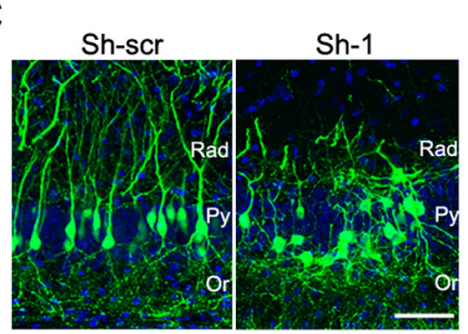

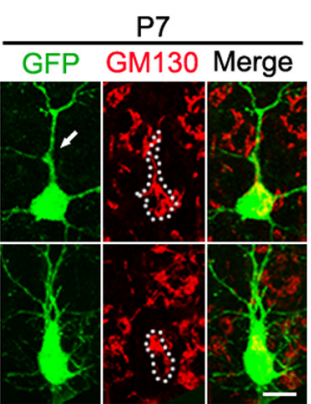

D

B

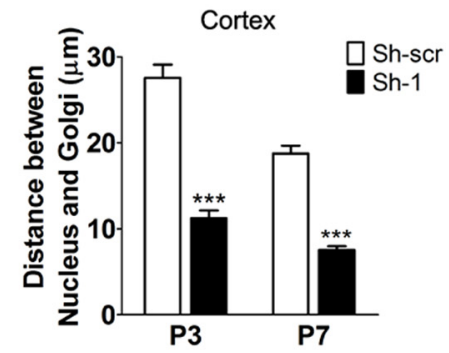

E

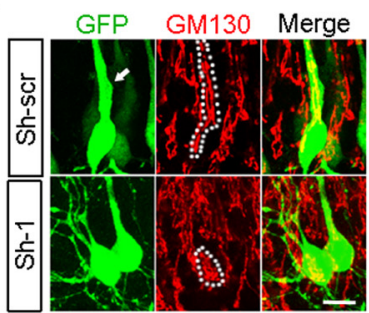

E

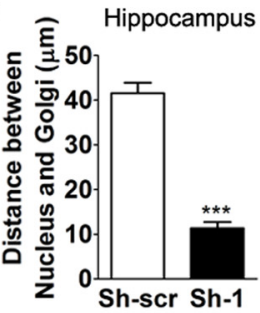

F
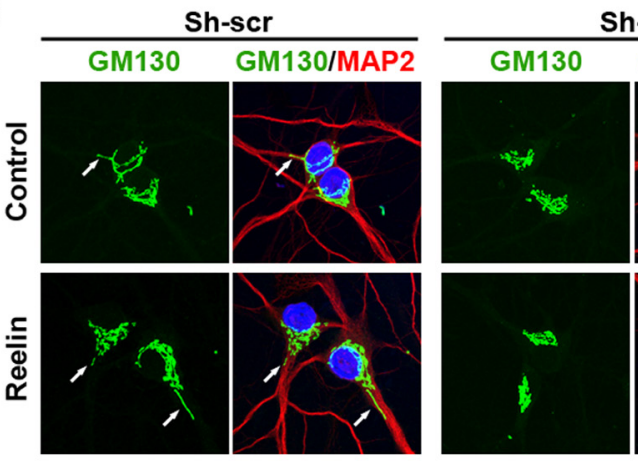

Sh-1
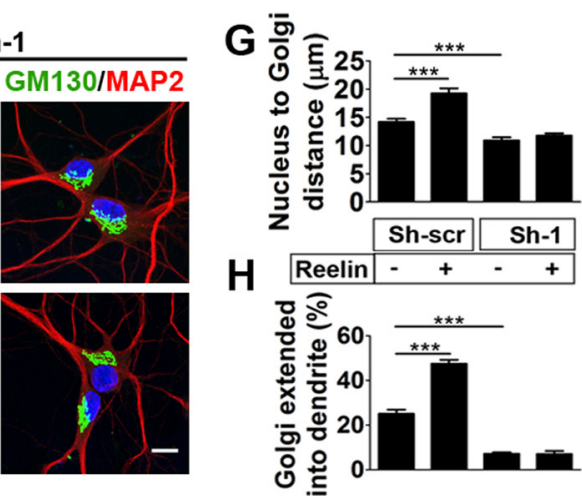

Figure 3. Ube3a knockdown disrupts the polarized distribution of the Golgi apparatus in pyramidal neurons. $A, C, D$, Representative images of layer II/III cortical neurons from P3 and P7 mouse brains $(\boldsymbol{A})$ and $C A 1$ neurons from P7 mouse brains $(\boldsymbol{C}, \boldsymbol{D})$ transfected with the indicated constructs. GM130 signals (red) outlined by white dots represent the Golgi apparatus of the corresponding GFP-labeled neuron. White arrows point to the proximal region of the apical dendrite. Scale bars: $A, D, 10 \mu \mathrm{m} ; \boldsymbol{C}, 50 \mu \mathrm{m}$. $\boldsymbol{B}, \boldsymbol{E}$, Quantitative analysis of the distance between the center of nucleus and the tip of the Golgi ribbon in cortical neurons $(\boldsymbol{B})$ and $C A 1$ neurons $(\boldsymbol{D}) . n=30-50$ neurons in each group. $\boldsymbol{F}$, Representative images of DIV6 hippocampal neurons infected with the indicated lentivirus. White arrows point to dendritic Golgi. $G, H$, Quantitative analysis of the distance between the center of nucleus and the tip of the Golgi ribbon (G) and MAP2-positive neuron ratio with Golgi extended into a dendrite $(\boldsymbol{H})$. Data represent mean \pm SEM. ${ }^{* * *} p<0.001$, statistically significant differences were determined using $t$ tests.

was only later on at P7, which corresponds to a period of exuberant dendrite morphogenesis, that the growth and arborization of the apical dendrite was selectively inhibited, ultimately resulting in a less polarized dendrite morphology. Moreover, pyramidal neurons in AS mice also presented reduced dendritic polarity with selective shortening of the apical dendrite (Fig. 4). Together, our findings highlight a novel mechanism for the development of the highly polarized dendritic arbor in pyramidal neurons, which occurs after radial migration. We also defined the developmental stage from P3 to P7 as a critical period for Ube3a-dependent polarized dendritic morphogenesis.

Consistent with a previous study performed in the visual cortex (Sato and Stryker, 2010), we observed a progressive enrichment of Ube3a in the nucleus as neurons matured both in vivo and in vitro (Fig. 2C). This spatial-temporal subcellular localization profile suggests that cytosolic Ube3a may account for the role of Ube3a in polarized dendrite outgrowth. Interestingly, the three mouse Ube3a isoforms presented distinct subcellular localization patterns in pyramidal neurons in vivo. Both Iso1 and Iso2 were primarily distributed within the cytosol and dendrites, whereas Iso3 was highly enriched in the nucleus (Fig. 2D). Among the three isoforms, only Iso2 was able to rescue the dendrite polarity defect caused by Ube3ashRNA expression (Fig. 2D). These findings support the notion that the three Ube3a isoforms have distinct functions and indicate that the catalytic function of Ube3a in cytosol is required for normal polarized dendritic morphogenesis. Our findings confirm those of previous studies indicating that Ube3a ubiquitin ligase activity is necessary for normal human cognitive function, and disruption of this activity leads to AS (Cooper et al., 2004).

We show here that, in Ube3a-shRNA-treated neurons with less polarized dendritic arbors, the Golgi apparatus failed to localize to the apical dendrite (Fig. 3). Importantly, at P3, when dendrite length and polarity indexes were comparable between shRNA and control groups, the Golgi in neurons treated with Ube3a-shRNA had displayed defect. The earlier onset of Golgi phenotype relative to dendrite polarity defect strongly suggests that Ube3A may regulate apical dendrite outgrowth and arborization by modulating the localization and function of the Golgi apparatus. In addition, our results point toward a cellautonomous requirement for Ube $3 a$ in regulating the polarized 
A

P8
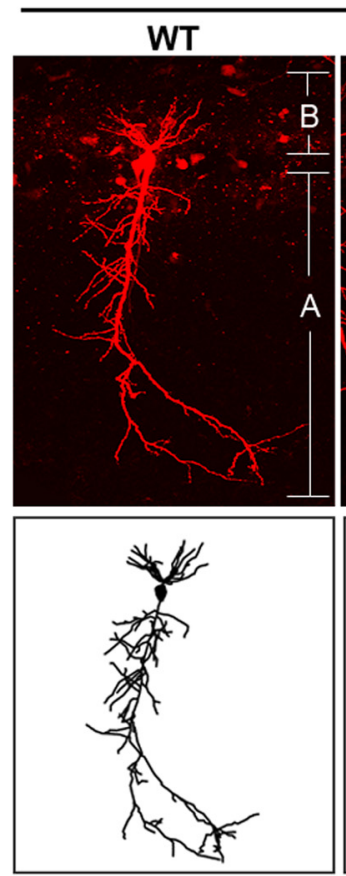

B

E
AS
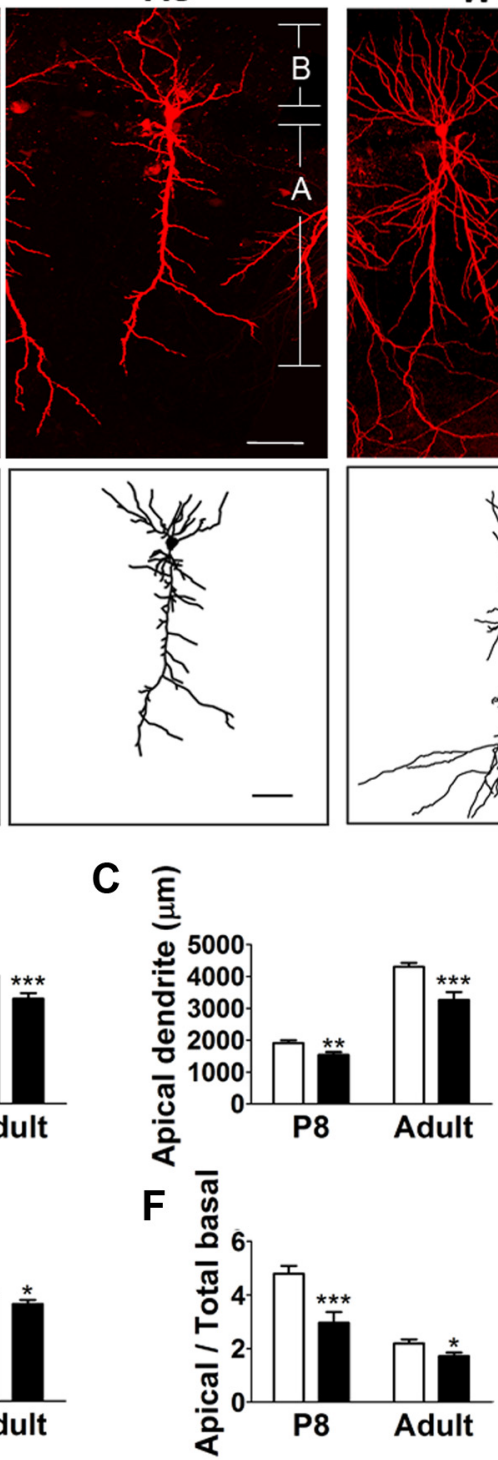

Adult
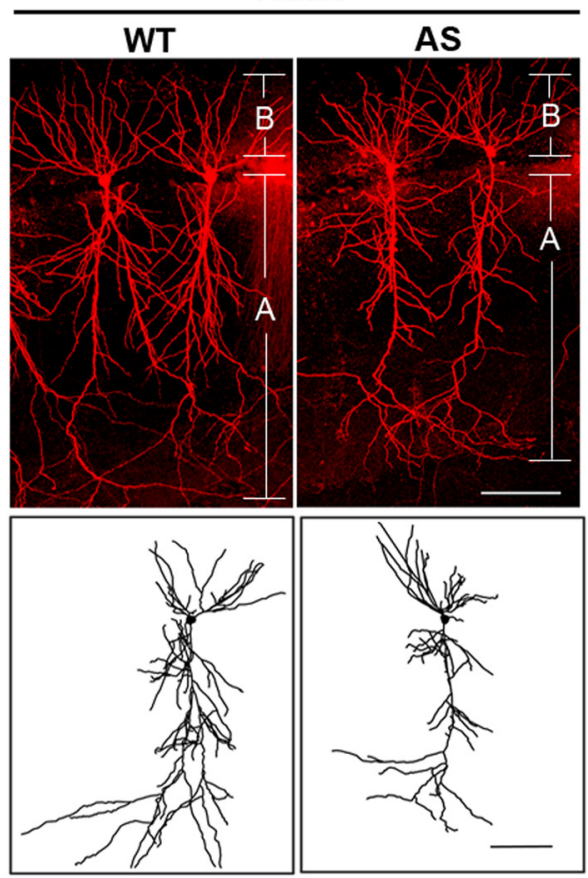
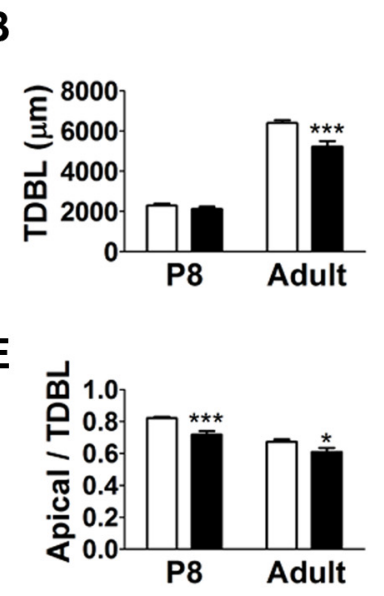

C $\overline{\mathrm{g}}$

$F$

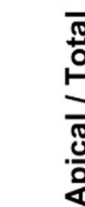

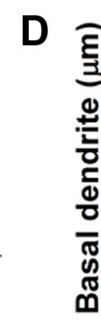

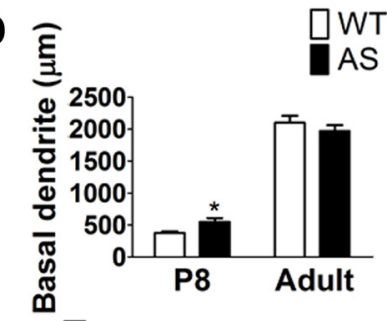

$\mathbf{G}$

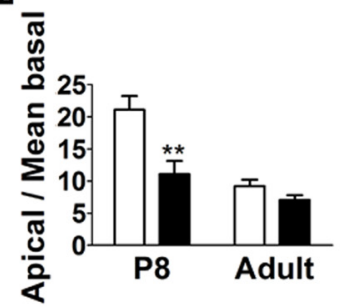

Figure 4. Pyramidal neurons in AS mice present impaired dendrite polarity with selective stunting of the apical dendrite. $\boldsymbol{A}$, Representative images (top) and tracings (bottom) of pyramidal neurons in the hippocampal CA1 region of 8-d-old and 3.5-month-old mice. Scale bar, $50 \mu \mathrm{m}$ for P8 neurons, $100 \mu \mathrm{m}$ for adult neurons. $\boldsymbol{B}-\boldsymbol{G}, \mathbf{Q}$ uantitative analyses of TDBL (B), apical dendrite length $(\boldsymbol{C})$, total basal dendrite length $(\boldsymbol{D})$, apical/TDBL ratio $(\boldsymbol{E})$, apical/total length of basal dendrites ratio $(\boldsymbol{F})$, and apical/basal dendrite mean length $(\boldsymbol{G})$. Data represent mean \pm SEM. P8, $n=$ 4 brains, total 15 neurons per group; adult, $n=3$ brains per group, 7 neurons per brain. ${ }^{*} p<0.05 ;{ }^{* *} p<0.01 ;{ }^{* * *} p<0.001$, statistically significant differences were determined using $t$ test.

distribution of the Golgi. Abnormal Golgi distribution patterns were only observed in neurons transfected with Ube3a-shRNA, while neighboring nontransfected neurons showed no similar defects.

Reelin-Dab1 signaling is essential for Golgi deployment into dendrite (Matsuki et al., 2010). It can induce a rapid reorganization of Golgi into dendrite in primary cultured hippocampal neurons. In our study, we reproduced its acute effect in vitro and found that downregulation of Ube3a significantly inhibited Golgi extension into dendrite and blocked Reelin-induced rapid deployment of Golgi into dendrite (Fig. $3 F-H$ ). This result suggests that Ube3a is indispensable for acute response of Golgi to Reelin. The evidence from some previous studies could help us to outline how Reelin and Ube3a regulate dendritic Golgi deployment. Reelin-Dab1 signaling controls Golgi morphology through antagonizing the action of LKB1-Stk25GM130 (Matsuki et al., 2010). The identification of several putative substrates and interacting partners of Ube3a that are associated with the Golgi apparatus, including Golgiassociated PDZ protein GOPC (Jeong et al., 2007), Golgi membrane associated protein GOLGA3 (Jung et al., 2005), Src family kinases (Oda et al., 1999) and TSC2 (Zheng et al., 2008), suggests a direct role for Ube3a in the structural and functional regulation of the Golgi apparatus. Based on these findings, it is likely that Ube3a and Reelin function in parallel and coordinately to regulate polarization of Golgi into dendrite. Alternatively, Ube3a may function downstream of Reelin to mediate its role in dendritic Golgi deployment. Further studies are needed to clarify how Ube3a regulates Golgi polarization in cooperation with Reelin signaling. 
In summary, our results indicate that cytoplasmic mouse Ube3a Iso2 is an important regulatory molecule for Golgidependent control of normal polarized dendrite development, and suggest that the impairment of dendrite polarity in pyramidal neurons caused by Ube3a depletion may lead to alterations in neural circuitry that underlie behavioral and cognitive dysfunctions associated with AS.

\section{References}

Cooper EM, Hudson AW, Amos J, Wagstaff J, Howley PM (2004) Biochemical analysis of Angelman syndrome-associated mutations in the E3 ubiquitin ligase E6-associated protein. J Biol Chem 279:41208-41217. CrossRef Medline

Demyanenko GP, Schachner M, Anton E, Schmid R, Feng G, Sanes J, Maness PF (2004) Close homolog of L1 modulates area-specific neuronal positioning and dendrite orientation in the cerebral cortex. Neuron 44: 423-437. CrossRef Medline

Dindot SV, Antalffy BA, Bhattacharjee MB, Beaudet AL (2008) The Angelman syndrome ubiquitin ligase localizes to the synapse and nucleus, and maternal deficiency results in abnormal dendritic spine morphology. Hum Mol Genet 17:111-118. Medline

Greer PL, Hanayama R, Bloodgood BL, Mardinly AR, Lipton DM, Flavell SW, Kim TK, Griffith EC, Waldon Z, Maehr R, Ploegh HL, Chowdhury S, Worley PF, Steen J, Greenberg ME (2010) The Angelman Syndrome protein Ube3A regulates synapse development by ubiquitinating arc. Cell 140:704-716. CrossRef Medline

Horton AC, Rácz B, Monson EE, Lin AL, Weinberg RJ, Ehlers MD (2005) Polarized secretory trafficking directs cargo for asymmetric dendrite growth and morphogenesis. Neuron 48:757-771. CrossRef Medline

Jan YN, Jan LY (2010) Branching out: mechanisms of dendritic arborization. Nat Rev Neurosci 11:316-328. CrossRef Medline

Jay V, Becker LE, Chan FW, Perry TL Sr (1991) Puppet-like syndrome of Angelman: a pathologic and neurochemical study. Neurology 41:416422. CrossRef Medline

Jeong KW, Kim HZ, Kim S, Kim YS, Choe J (2007) Human papillomavirus type $16 \mathrm{E} 6$ protein interacts with cystic fibrosis transmembrane regulatorassociated ligand and promotes E6-associated protein-mediated ubiquitination and proteasomal degradation. Oncogene 26:487-499. CrossRef Medline

Jiang YH, Armstrong D, Albrecht U, Atkins CM, Noebels JL, Eichele G, Sweatt JD, Beaudet AL (1998) Mutation of the Angelman ubiquitin ligase in mice causes increased cytoplasmic p53 and deficits of contextual learning and long-term potentiation. Neuron 21:799-811. CrossRef Medline

Jung SY, Malovannaya A, Wei J, O'Malley BW, Qin J (2005) Proteomic analysis of steady-state nuclear hormone receptor coactivator complexes. Mol Endocrinol 19:2451-2465. CrossRef Medline

Lu Y, Wang F, Li Y, Ferris J, Lee JA, Gao FB (2009) The Drosophila homo- logue of the Angelman syndrome ubiquitin ligase regulates the formation of terminal dendritic branches. Hum Mol Genet 18:454-462. Medline

Mabb AM, Judson MC, Zylka MJ, Philpot BD (2011) Angelman syndrome: insights into genomic imprinting and neurodevelopmental phenotypes. Trends Neurosci 34:293-303. CrossRef Medline

Margolis SS, Salogiannis J, Lipton DM, Mandel-Brehm C, Wills ZP, Mardinly AR, Hu L, Greer PL, Bikoff JB, Ho HY, Soskis MJ, Sahin M, Greenberg ME (2010) EphB-mediated degradation of the RhoA GEF Ephexin 5 relieves a developmental brake on excitatory synapse formation. Cell 143:442-455. CrossRef Medline

Matsuki T, Matthews RT, Cooper JA, van der Brug MP, Cookson MR, Hardy JA, Olson EC, Howell BW (2010) Reelin and stk25 have opposing roles in neuronal polarization and dendritic Golgi deployment. Cell 143:826-836. CrossRef Medline

Nakahira E, Yuasa S (2005) Neuronal generation, migration, and differentiation in the mouse hippocampal primoridium as revealed by enhanced green fluorescent protein gene transfer by means of in utero electroporation. J Comp Neurol 483:329-340. CrossRef Medline

Oda H, Kumar S, Howley PM (1999) Regulation of the Src family tyrosine kinase Blk through E6AP-mediated ubiquitination. Proc Natl Acad Sci U S A 96:9557-9562. CrossRef Medline

Saito T, Nakatsuji N (2001) Efficient gene transfer into the embryonic mouse brain using in vivo electroporation. Dev Biol 240:237-246. CrossRef Medline

Sasaki Y, Cheng C, Uchida Y, Nakajima O, Ohshima T, Yagi T, Taniguchi M, Nakayama T, Kishida R, Kudo Y, Ohno S, Nakamura F, Goshima Y (2002) Fyn and Cdk5 mediate semaphorin-3A signaling, which is involved in regulation of dendrite orientation in cerebral cortex. Neuron 35:907-920. CrossRef Medline

Sato M, Stryker MP (2010) Genomic imprinting of experience-dependent cortical plasticity by the ubiquitin ligase gene Ube3a. Proc Natl Acad Sci U S A 107:5611-5616. CrossRef Medline

Tabata H, Nakajima K (2002) Neurons tend to stop migration and differentiate along the cortical internal plexiform zones in the Reelin signaldeficient mice. J Neurosci Res 69:723-730. CrossRef Medline

Whitford KL, Dijkhuizen P, Polleux F, Ghosh A (2002) Molecular control of cortical dendrite development. Annu Rev Neurosci 25:127-149. CrossRef Medline

Yashiro K, Riday TT, Condon KH, Roberts AC, Bernardo DR, Prakash R, Weinberg RJ, Ehlers MD, Philpot BD (2009) Ube3a is required for experience-dependent maturation of the neocortex. Nat Neurosci 12: 777-783. CrossRef Medline

Zheng L, Ding H, Lu Z, Li Y, Pan Y, Ning T, Ke Y (2008) E3 ubiquitin ligase E6AP-mediated TSC2 turnover in the presence and absence of HPV16 E6. Genes Cells 13:285-294. CrossRef Medline

Zheng W, Geng AQ, Li PF, Wang Y, Yuan XB (2012) Robo4 regulates the radial migration of newborn neurons in developing neocortex. Cereb Cortex 22:2587-2601. CrossRef Medline 